\section{PROTEÍNA, LISINA Y TRIPTÓFANO EN POBLACIONES NATIVAS DE MAÍZ MIXTECO}

\section{PROTEIN, LYSINE AND TRYPTOPHAN IN NATIVE POPULATIONS OF MIXTECO MAIZE}

\author{
Araceli M. Vera-Guzmán ${ }^{1 \star}$, J. Luis Chávez-Servia ${ }^{1}$ \\ y José C. Carrillo-Rodríguez ${ }^{2}$
}

${ }^{1}$ Instituto Politécnico Nacional, CIIDIR-Unidad Oaxaca, Hornos \#1003, 71230, Santa Cruz Xoxocotlán, Oaxaca, México. ${ }^{2}$ Instituto Tecnológico del Valle de Oaxaca, Ex-Hacienda Nazareno. 71230, Santa Cruz Xoxocotlán, Oaxaca, México.

${ }^{*}$ Autor para correspondencia (avera@ipn.mx)

\section{RESUMEN}

La diversidad de variantes locales de maíz (Zea mays L.) en la Mixteca Oaxaqueña, México, es una respuesta a las presiones de selección natural y humana. Con el objetivo de evaluar el contenido de proteína, lisina y triptófano en el maíz nativo de la Mixteca, se hizo una colecta de $\mathbf{7 0}$ muestras poblacionales en $\mathbf{5 1}$ comunidades de agricultores de $\mathbf{1 3}$ municipios del distrito geopolítico de Tlaxiaco, Oaxaca. Las muestras fueron sembradas en el ciclo primavera-verano de 2008, bajo un diseño experimental de bloques completos al azar con cuatro repeticiones, en San Martín Huamelulpam. En la cosecha se tomó aleatoriamente una muestra de $300 \mathrm{~g}$ de grano por parcela experimental y en cada una se cuantificó el contenido de proteína, lisina y triptófano, por triplicado. Se observaron diferencias $(P \leq 0.05)$ en el contenido de proteína, lisina y triptófano del grano, entre y dentro de grupos de colectas de maíz Mixteco de diferente color de grano (amarillo, azul, blanco, rojo y pinto). En proteína, 10 colectas superaron al testigo tipo QPM. En lisina y proteína no hubo diferencias significativas entre el testigo y las colectas de grano amarillo, blanco rojo y pinto evaluadas. La colecta CIIDIR-185 se detectó como promisoria debido a que fue estadísticamente igual al testigo en triptófano y lisina y lo superó en contenido de proteína.

Palabras clave: Zea mays, aminoácidos, composición de grano, variación fenotípica.

\section{SUMMARY}

The landrace diversity of maize (Zea mays L.) in the Mixteca Oaxaqueña region in México is a response to natural and human selection pressures. In order to evaluate the protein, lysine and tryptophan content in the native Mixteca maizes, a collection of 70 population samples at 51 farmer communities belonging to 13 municipalities of the Tlaxiaco, Oaxaca district was determined. Populations were planted during the 2008 Spring - Summer agricultural cycle under a complete randomized blocks design with four replications at San Martín Huamelulpam. At harvest, a random $300 \mathrm{~g}$ kernel sample per experimental plot was taken and the protein, lysine and tryptophan contents were assessed. Significant differences $(P \leq 0.05)$ were observed among and within groups of accessions of Mixteca maizes with different kernel color (yellow, blue, white, red and color combinations) for the protein, lysine and tryptophan content in the whole kernel. Control and evalua- ted accessions of yellow, white, red kernel and color combinations of kernel color showed no significant differences in lysine and tryptophan content. In protein, 10 accessions surpassed the QPM control. Accession CIIDIR-185 is promissory since it presented similar values in tryptophan and lysine, and higher values in protein content in relation to the control.

Index words: Zea mays, amino acids, kernel composition, phenotypic variation.

\section{INTRODUCCIÓN}

En el Estado de Oaxaca se cosechan anualmente más de 500 mil hectáreas de maíz (Zea mays L.), y en la región Mixteca alrededor de 120 mil hectáreas; del total, $87.6 \%$ corresponden a siembra de temporal (secano) en monocultivo o en asociación con otros cultivos, con un rendimiento promedio en la Mixteca de 0.4 a $2.7 \mathrm{t} \mathrm{ha}^{-1}$ (OEIDRUS, 2010). El maíz se cultiva en la Mixteca desde tiempos precolombinos (Anderson y Finan, 1945) y prevalece en el sistema "milpa", donde se reconocen diferentes colores de grano como azul, rojo, blanco, amarillo o pinto y se asocia, doble o triple, con frijol (Phaseolus vulgaris L.), calabaza (Cucurbita pepo L.), chilacayote (Cucurbita fiscifolia L.) o haba (Vicia faba L.) (Chávez y Diego, 2011).

En la Mixteca oaxaqueña, el ambiente y selección humana ejercen fuertes presiones sobre las poblaciones cultivadas de maíz hasta definir poblaciones con adaptaciones específicas, como al estrés hídrico en los maíces de "cajete" (Muñoz et al., 2002; Hayano-Kanashiro et al., 2009), cambios en morfología de planta y mazorca distintivas como en la raza Mixteco, denominada por Benz (1997) y en diversos grupos poblacionales o complejos raciales (Chávez-Servia et al., 2011; Chávez y Diego, 2011). También es de esperar que las fuertes presiones de selección humana y natural hayan influenciado la composición del grano en los maíces cultivados en diferentes micronichos de la Mixteca, ya que se cultivan en suelos de baja fertilidad, ligeros, de alta erosión y con bajo o nulo uso de insumos (Contreras-Hinojosa et al., 2005).

En particular, la selección que hacen los agricultores de caracteres fisiológicos de planta y mazorca han determinado, en parte, las poblaciones de maíz que hoy en día se observan en las parcelas de cultivo (Soleri y Cleveland, 2001; Pressoir y Berthaud, 2004). En las comunidades rurales, el maíz es la base de la alimentación, y mediante procesos básicos de transformación, como la nixtamalización, convierten al grano en diversos productos y platillos gastronómicos entre los que se destacan las tortillas de colores distintos, tamales, pozole, totopos, atoles, etc. Sin embargo, la calidad nutritiva del grano de los maíces nativos, particularmente los cultivados en la Mixteca, no ha sido completamente identificada, evaluada y caracterizada, con el propósito de 
promover la diversidad de maíces que mejoren la dieta y fortalezcan la salud de las comunidades indígenas.

En México el maíz es la principal fuente de energía y proteínas de la población. En particular, los maíces nativos aportan a la dieta compuestos antioxidantes (López-Martínez et al., 2009; Salinas-Moreno et al., 2003), carotenoides (Lozano-Alejo et al., 2007), aminoácidos (Vidal et al., 2008) y diversos elementos nutricionales que tienen un efecto positivo en la prevención de enfermedades como hipertensión, altos niveles de triglicéridos, colesterol (Guzmán-Gerónimo et al., 2011; Com. pers. ${ }^{1}$ ) y efecto anticarcinogénico colorrectal (Hawigara et al., 2001). Bajo este contexto, el objetivo de este trabajo fue evaluar el contenido de proteína, lisina y triptófano en una colección de 70 muestras poblacionales de maíz nativo de la Mixteca oaxaqueña.

\section{MATERIALES Y METÓDOS}

\section{Material biológico}

Se colectaron 70 muestras poblacionales de maíz durante los meses de febrero-abril de 2008 a una altitud de 2000 a $2900 \mathrm{~m}$ en 51 comunidades de agricultores de 13 municipios de la Mixteca oaxaqueña: Chalcatongo de Hidalgo, San Juan Ñumí, Santa Catarina Ticuá, San Miguel el Grande, Santa Cruz Nundaco, San Antonio Nduaxico, San Esteban Atatlahuca, Santa Catarina Yosonotú, San Pedro Mártir Yucuxaco, Santa Cruz Tayata, Santiago Nundiche, San Pedro Molinos y Magdalena Peñasco. Las muestras de las colectas se clasificaron por color de grano: azul, blanco, rojo y pinto (dos o más combinaciones de granos azules, rojos, blancos, amarillos, etc., en la misma mazorca), y se sembraron en una parcela de San Martín Huamelúlpam, Oaxaca, durante el ciclo primavera-verano 2008, bajo un diseño de bloques completos al azar con cuatro repeticiones. A la cosecha se tomaron muestras aleatorias de $300 \mathrm{~g}$ de grano de cada unidad experimental y se refrigeraron por 10 meses en frascos ámbar hasta su análisis. Se utilizó un testigo externo (S.L. 2009, var. 'VG 1-2', del Instituto Nacional de Investigaciones Forestales, Agrícolas y Pecuarias) del tipo QPM (maíz de alta calidad proteínica, por sus siglas en inglés).

\section{Composición de grano}

El grano de maíz se molió en un molino ciclónico y se cribó por un tamiz con malla de $500 \mu \mathrm{m}$. La muestra mo-

\footnotetext{
${ }^{1}$ Guzmán-Gerónimo R I, T M Alarcón-Zavala, E Silva-Hernández, J E Meza-Alvarado, S Herrerra-Meza, R M Oliart-Ros, J L ChávezServia (2011) Polifenoles totales, antocianinas, actividad antioxidante de maíz azul y su efecto en ratas Wistar con síndrome metabólico inducido. In: 3er. Congreso Internacional de Biología, Química y Agronomía, Septiembre 28-Octubre 1, 2011. Universidad de Guadalajara. Guadalajara, México.
}

lida se desengrasó con acetona de acuerdo con el método propuesto por Obi (1982). Posteriormente, a cada muestra se le determinó el contenido de proteínas por el método de Bradford (1976), técnica que se basa en la interacción del indicador azul brillante de Coomassie G-250 con las proteínas. La cuantificación de proteína se hizo por medio de un espectrofotómetro (Shimadzu, modelo UV-1601®; Kyoto, Japan) y con base en la curva de calibración de albúmina de suero de bovino ( $98 \%$ de pureza; Sigma), como estándar de proteína. Los resultados se reportan en porcentaje de proteína en base húmeda libre de aceite (p.b.h.) y en base seca libre de aceite (p.b.s.). El triptófano y lisina en base seca se cuantificaron de acuerdo con los métodos colorimétricos descritos por Villegas et al. (1985) y se expresaron en porcentaje. Todos los análisis se hicieron por triplicado.

\section{Análisis estadístico}

Las diferencias entre grupos de color de grano y poblaciones dentro de grupos se evaluaron mediante un análisis de varianza con un modelo lineal de bloques completos al azar y una prueba de comparación de medias de Tukey ( $\mathrm{P}$ $\leq 0.05)$. Con los promedios estandarizados por muestra se hizo un análisis de componentes principales para describir los patrones poblacionales con referencia al testigo QPM, todo ello mediante el paquete estadístico SAS versión 8.0 (SAS Institute, 1999).

\section{RESULTADOS Y DISCUSIÓN}

Los resultados aquí presentados se refieren a los contenidos de proteína lisina y triptófano del grano completo, sin separar endospermo ni germen. En el análisis de varianza se detectaron diferencias $(P \leq 0.01)$ entre y dentro grupos de colectas de diferente color de grano. Se observó mayor variación en contenido de proteína entre grupos que dentro de grupos (Cuadro 1).

En los trabajos previos de caracterización morfológica realizados por Chávez-Servia et al. (2011) en las mismas muestras de este estudio, se observaron diferencias significativas en características de mazorca y grano. Aunque no son los mismos patrones diferenciales, también se reflejaron diferencias sustanciales de muestra a muestra en contenido de proteína, lisina y triptófano. En los promedios por grupo de color de grano, se observó que los maíces denominados como pintos y rojos fueron semejantes en composición al testigo (QPM), y al valor medio registrado por Sierra et al. (2011) en el híbrido 'H-564C' de alta calidad de proteína recientemente liberado para el trópico; y sobrepasan ligeramente al promedio reportado por Vidal et al. (2008) para los maíces nativos de Nayarit (Cuadros 2, 3 y 4).

En relación con el contenido de proteína base húmeda y 
Cuadro 1. Cuadrados medios del análisis de varianza, coeficientes de variación y promedios de proteína, triptófano y lisina de 70 poblaciones de maíz de la Mixteca oaxaqueña.

\begin{tabular}{lcccc}
\hline Fuente de variación & $\begin{array}{c}\text { Proteína en base } \\
\text { húmeda }(\%)\end{array}$ & $\begin{array}{c}\text { Proteína en base } \\
\text { seca }(\%)\end{array}$ & $\begin{array}{c}\text { Triptófano en base } \\
\text { seca }(\%)\end{array}$ & $\begin{array}{c}\text { Lisina en base } \\
\text { seca }(\%)\end{array}$ \\
\hline Grupo de color de grano & $7.51^{* *}$ & $9.24^{* *}$ & $<0.001^{* *}$ & $<0.001^{* *}$ \\
Colecta (grupo) & $6.61^{* *}$ & $8.49^{* *}$ & $<0.001^{* *}$ & $<0.001^{* *}$ \\
Coeficiente de variación (\%) & 4.99 & 4.99 & 3.740 & 3.740 \\
Promedio & 10.99 & 12.33 & 0.067 & 0.392 \\
Testigo QPM & 12.30 & 13.80 & 0.090 & 0.620 \\
\hline
\end{tabular}

${ }^{\star *}$ Diferencias significativas $(\mathrm{P} \leq 0.01)$.

seca, 32 colectas se comportaron estadísticamente iguales al testigo QPM y 11 de ellas sobrepasaron al valor promedio estimado para el testigo en $>12.3 \%$ de proteína en base húmeda y $>13.8 \%$ de proteína en base seca. Un comportamiento similar reportaron Coutiño et al. (2008) en las variedades 'V-229' y 'V-231A', Vidal et al. (2008) en colectas de maíz nativo de Nayarit y Vázquez et al. (2010) en maíces nativos de Hidalgo, lo que indica que algunas poblaciones nativas o material mejorado a partir de los acervos genéticos locales del maíz mexicano, tienen igual o mayor contenido de proteína total que los denominados QPM; no obstante, estos últimos pueden tener mayores proporciones de algunos aminoácidos esenciales. Es de primordial importancia señalar que la composición del grano está influenciada por las condiciones de cultivo o por efectos considerados como no-genéticos.

Respecto al contenido de triptófano en base seca, se observó que ninguna de las colectas superó al valor promedio determinado para el testigo QPM (0.09 \% de triptófano); sin embargo, la colecta CIIDIR-185 no mostró diferencias significativas con respecto al testigo ( $>0.08 \%)$. Este mismo efecto observó Sierra et al. (2011) al comparar el híbrido ' $\mathrm{H}-564 \mathrm{C}$ ' de alta calidad de proteína con otras variedades mejoradas ('V-556AC'). No obstante, se observó un efecto estabilizador del alto contenido de triptófano en los materiales mejorados con el objetivo de incrementar la calidad de proteína; por lo que no se deben dejar de lado los acervos genéticos locales que presentan esa característica, como también se confirmó en el estudio de Vidal et al. (2008). Además, no sólo se detectaron materiales promisorios en grano blanco sino que también los hay en azul, amarillo, rojo y pinto.

Los valores promedio de 0.4 a $0.6 \%$ de lisina en base seca de 29 colectas fueron estadísticamente iguales al valor determinado para el testigo QPM (0.62 \%). Estos valores fueron ligeramente superiores a los reportados para el híbrido
'H-564C' (Sierra et al., 2011), al estimado para las variedades 'V-229' y 'V-231A' de la raza Comiteco reportadas por Coutiño et al. (2008) y al promedio de los maíces nativos de Nayarit (Vidal et al., 2008). Esto puede indicar, en parte, que el ambiente de cultivo influye de manera significativa en la expresión del carácter y también refleja una estimación del estado de la composición de grano y no necesariamente que existan genes en las poblaciones que le confieren esa característica (Mittelmann et al., 2011). Esto conlleva a pensar que deben obtenerse diferentes estimaciones de la composición del grano en colectas cultivadas en diferentes ambientes para obtener una estimación del efecto ambiental y del efecto genotípico.

En el análisis de componentes principales se determinó que en los dos primeros componentes se concentró $89.6 \%$ de la variabilidad total evaluada; por consiguiente, pueden considerarse a los dos primeros componentes principales como índices de selección. En la Figura 1 se ubicó al testigo QPM en el cuadrante inferior derecho y varias colectas en el cuadrante superior derecho. Este patrón indica que en la evaluación se observaron más de 10 colectas que superan al testigo en proteína, pero no en contenido de lisina y triptófano.

\section{CONCLUSIONES}

Se detectaron diferencias significativas entre y dentro de grupos de colectas de maíz Mixteco de diferente color de grano (amarillo, azul, blanco, rojo y pinto), en el contenido de proteína, lisina y triptófano del grano entero. En proteína en base húmeda y proteína en base seca, 11 colectas superaron al testigo QPM. En lisina 29 colectas fueron superiores al testigo . La colecta CIIDIR-185 se considera promisoria porque fue estadísticamente igual al testigo QPM en triptófano y lisina, y lo superó en contenido de proteína. 
Cuadro 2. Contenido promedio de proteína en base húmeda y en base seca, triptófano y lisina de 70 poblaciones de maíz de la Mixteca de Oaxaca de color amarillo y azul.

\begin{tabular}{|c|c|c|c|c|c|}
\hline Colecta & $\begin{array}{l}\text { Origen de la colecta (comunidad o agencia, } \\
\text { municipio) }\end{array}$ & $\begin{array}{l}\text { Proteína base } \\
\text { húmeda }(\%)\end{array}$ & $\begin{array}{l}\text { Proteína base } \\
\text { seca }(\%)\end{array}$ & $\begin{array}{l}\text { Triptófano } \\
\text { base seca (\%) }\end{array}$ & $\begin{array}{l}\text { Lisina base } \\
\text { seca }(\%)\end{array}$ \\
\hline \multicolumn{6}{|c|}{ Colectas de grano amarillo } \\
\hline CIIDIR-59 & Cañada María, Tlaxiaco & 10.5 & 11.9 & 0.063 & 0.313 \\
\hline CIIDIR-50 & El Uval, Santa Cruz Tayata & 9.7 & 10.9 & 0.063 & 0.280 \\
\hline CIIDIR-11 & Guadalupe Mirasol, San Juan Ñumí & 10.5 & 11.7 & 0.068 & 0.380 \\
\hline CIIDIR-78 & Cabacúa, Magdalena Peñasco & 10.1 & 11.4 & 0.063 & 0.373 \\
\hline CIIDIR-94 & Yosocahua, Magdalena Peñasco & 10.1 & 11.2 & 0.068 & 0.420 \\
\hline CIIDIR-102 & Chapultepec, Chacaltongo de Hidalgo & 10.5 & 11.9 & 0.069 & 0.330 \\
\hline CIIDIR-113 & Abasolo, Chacaltongo de Hidalgo & 11.9 & 13.4 & 0.081 & 0.496 \\
\hline CIIDIR-115 & Reforma, Chacaltongo de Hidalgo & 8.7 & 9.8 & 0.055 & 0.420 \\
\hline CIIDIR-136 & Reforma, Chacaltongo de Hidalgo & 10.4 & 11.7 & 0.064 & 0.586 \\
\hline CIIDIR-198 & Cañada de Morelos, Chacaltongo de Hidalgo & 12.8 & 14.4 & 0.075 & 0.526 \\
\hline CIIDIR-196 & Ignacio Zaragoza, San Miguel El Grande & 10.5 & 11.8 & 0.068 & 0.303 \\
\hline CIIDIR-187 & Yucuiji, San Esteban Atatlahuca & 11.1 & 12.5 & 0.077 & 0.496 \\
\hline CIIDIR-177 & Morelos, San Esteban Atatlahuca & 12.6 & 14.1 & 0.076 & 0.386 \\
\hline CIIDIR-175 & Morelos, San Esteban Atatlahuca & 9.9 & 11.1 & 0.072 & 0.506 \\
\hline CIIDIR-166 & Ignacio Zaragoza, San Miguel El Grande & 11.7 & 13.3 & 0.066 & 0.356 \\
\hline CIIDIR-165 & Ignacio Zaragoza, San Miguel El Grande & 10.5 & 11.8 & 0.061 & 0.476 \\
\hline CIIDIR-153 & Yute Tintaku, San Esteban Atatlahuca & 9.9 & 10.9 & 0.068 & 0.313 \\
\hline \multirow[t]{2}{*}{ CIIDIR-147 } & Benito Juárez, San Miguel El Grande & 9.7 & 11.0 & 0.059 & 0.323 \\
\hline & Promedio de grupo & $10.6 c^{\dagger}$ & $11.9 \mathrm{c}$ & $0.068 \mathrm{ab}$ & $0.404 \mathrm{a}$ \\
\hline \multicolumn{6}{|c|}{ Colectas de grano azul } \\
\hline CIIDIR-197 & Ignacio Zaragoza, San Miguel El Grande & 10.4 & 11.6 & 0.068 & 0.426 \\
\hline CIIDIR-190 & Yucuiji, San Esteban Atatlahuca & 12.1 & 13.6 & 0.077 & 0.276 \\
\hline CIIDIR-185 & Ojo de Agua, Santa Cruz Nundaco & 14.2 & 15.9 & 0.086 & 0.533 \\
\hline CIIDIR-163 & Ignacio Zaragoza, San Miguel el Grande & 8.3 & 9.3 & 0.059 & 0.326 \\
\hline CIIDIR-129 & Independencia, Chalcatongo de Hidalgo & 10.2 & 11.3 & 0.068 & 0.356 \\
\hline CIIDIR-112 & Abasolo, Chalcatongo de Hidalgo & 10.8 & 12.1 & 0.055 & 0.230 \\
\hline CIIDIR-107 & Abasolo, Chalcatongo de Hidalgo & 10.7 & 12.0 & 0.056 & 0.296 \\
\hline CIIDIR-12 & Guadalupe Mirasol, San Juan Ñumí & 9.6 & 10.9 & 0.056 & 0.276 \\
\hline CIIDIR-05 & San Nicolás, Tlaxiaco & 12.3 & 13.7 & 0.069 & 0.310 \\
\hline CIIDIR-02 & San Esteban, San Esteban Atatlahuca & 9.9 & 11.1 & 0.061 & 0.473 \\
\hline \multirow[t]{2}{*}{ CIIDIR-125 } & Independencia, Chalcatongo de Hidalgo & 8.9 & 9.9 & 0.063 & 0.323 \\
\hline & Promedio de grupo & $10.6 \mathrm{c}$ & $11.9 \mathrm{c}$ & $0.065 \mathrm{c}$ & $0.348 \mathrm{~b}$ \\
\hline
\end{tabular}

${ }^{ \pm}$En cada columna, medias de grupos con letras iguales no son estadísticamente diferentes (Tukey, 0.05). 
Cuadro 3. Contenido promedio de proteína base en húmeda y en base seca, triptófano y lisina de 70 poblaciones de maíz de la Mixteca de Oaxaca de color blanco y rojo.

\begin{tabular}{|c|c|c|c|c|c|}
\hline Colecta & $\begin{array}{l}\text { Origen de la colecta (comunidad o agen- } \\
\text { cia, municipio) }\end{array}$ & $\begin{array}{l}\text { Proteína base } \\
\text { húmeda }(\%)\end{array}$ & $\begin{array}{l}\text { Proteína base } \\
\quad \text { seca }(\%)\end{array}$ & $\begin{array}{c}\text { Triptófano } \\
\text { base seca (\%) }\end{array}$ & $\begin{array}{l}\text { Lisina base } \\
\text { seca }(\%)\end{array}$ \\
\hline \multicolumn{6}{|c|}{ Colectas de grano blanco } \\
\hline CIIDIR-182 & La Loma, Santa Cruz Nundaco & 9.8 & 10.8 & 0.058 & 0.316 \\
\hline CIIDIR-77 & Barrio San Jose, Santa Catarina Ticua & 9.6 & 10.7 & 0.058 & 0.466 \\
\hline CIIDIR-76 & Chamizal, Santa Catarina Ticua & 10.7 & 12.1 & 0.067 & 0.460 \\
\hline CIIDIR-75 & Fortín Juárez, Santa Catarina Ticua & 10.2 & 11.5 & 0.066 & 0.373 \\
\hline CIIDIR-70 & Barrio Guadalupe, San Pedro Molinos & 11.5 & 13.1 & 0.071 & 0.490 \\
\hline CIIDIR-68 & Morelos, Santiago Nundiche & 8.5 & 9.7 & 0.062 & 0.530 \\
\hline CIIDIR-57 & Enduayavi, Santa Cruz Tayata & 10.3 & 11.6 & 0.055 & 0.260 \\
\hline CIIDIR-149 & Benito Juárez, San Miguel El Grande & 12.2 & 13.6 & 0.071 & 0.416 \\
\hline CIIDIR-157 & Yutebey, San Esteban Atatlahuca & 9.7 & 10.9 & 0.067 & 0.476 \\
\hline CIIDIR-178 & Morelos, San Esteban Atatlahuca & 9.5 & 10.4 & 0.062 & 0.346 \\
\hline CIIDIR-42 & Cañada Maria, San Pedro Mártir Yucuxaco & 11.1 & 12.3 & 0.067 & 0.366 \\
\hline CIIDIR-39 & Cañada Maria, San Pedro Mártir Yucuxaco & 12.5 & 14.2 & 0.070 & 0.570 \\
\hline CIIDIR-34 & Río, San Juan Ñumí, & 11.9 & 13.4 & 0.071 & 0.346 \\
\hline CIIDIR-27 & San Jose Ñumí, San Juan Ñumi & 8.7 & 9.7 & 0.073 & 0.370 \\
\hline CIIDIR-19 & Santo Domingo Yosoñama, San Juan Ñumi & 12.3 & 13.9 & 0.069 & 0.373 \\
\hline CIIDIR-14 & San Antonio Nduaxico, San Juan Ñumi & 11.8 & 13.3 & 0.063 & 0.376 \\
\hline CIIDIR-04 & Barrio San Nicolas, Tlaxiaco & 9.9 & 10.9 & 0.067 & 0.473 \\
\hline CIIDIR-137 & Iturbide, San Miguel El Grande & 13.5 & 15.3 & 0.069 & 0.380 \\
\hline CIIDIR-134 & Reforma, Chacaltongo de Hidalgo & 10.3 & 11.5 & 0.067 & 0.500 \\
\hline CIIDIR-132 & Allende, Chacaltongo de Hidalgo & 13.3 & 14.9 & 0.069 & 0.336 \\
\hline CIIDIR-126 & Independencia, Chacaltongo de Hidalgo & 11.2 & 12.8 & 0.071 & 0.346 \\
\hline \multirow[t]{2}{*}{ CIIDIR-109 } & Abasolo, Chacaltongo de Hidalgo & 12.3 & 13.8 & 0.072 & 0.526 \\
\hline & Promedio de grupo & $10.9 \mathrm{bc}$ & $12.3 \mathrm{bc}$ & $0.067 \mathrm{bc}$ & $0.413 \mathrm{a}$ \\
\hline \multicolumn{6}{|c|}{ Colectas de grano rojo } \\
\hline CIIDIR-183 & La Loma, Santa Cruz Nundaco & 11.0 & 12.4 & 0.067 & 0.340 \\
\hline CIIDIR-158 & Yutebey, San Esteban Atatlahuca & 12.7 & 14.4 & 0.061 & 0.373 \\
\hline CIIDIR-160 & Ignacio Zaragoza, San Miguel El Grande & 9.3 & 10.5 & 0.073 & 0.363 \\
\hline CIIDIR-173 & Yuñana, San Esteban Atatlahuca & 11.4 & 12.9 & 0.069 & 0.356 \\
\hline CIIDIR-176 & Morelos, San Esteban Atatlahuca & 13.8 & 15.3 & 0.074 & 0.403 \\
\hline CIIDIR-194 & Santa Catarina Yosonotú & 11.4 & 12.8 & 0.065 & 0.480 \\
\hline CIIDIR-195 & Ignacio Zaragoza, San Miguel El Grande & 9.3 & 10.4 & 0.071 & 0.386 \\
\hline CIIDIR-13 & Guadalupe Mirasol, San Juan Ñumí & 9.3 & 10.5 & 0.062 & 0.576 \\
\hline CIIDIR-41 & Cañada María, San Pedro Mártir Yucuxaco & 9.1 & 10.2 & 0.065 & 0.306 \\
\hline CIIDIR-122 & Fortín Juárez, Santa Catarina Ticúa & 11.9 & 13.4 & 0.065 & 0.393 \\
\hline CIIDIR-103 & Chapultepec, Chacaltongo de Hidalgo & 14.2 & 15.9 & 0.081 & 0.480 \\
\hline \multirow[t]{2}{*}{ CIIDIR-186 } & Santa Cruz Nundaco & 11.2 & 12.5 & 0.067 & 0.280 \\
\hline & Promedio de grupo & $11.2 \mathrm{~b}$ & $12.6 \mathrm{~b}$ & $0.068 \mathrm{a}$ & $0.395 \mathrm{a}$ \\
\hline
\end{tabular}

${ }^{ \pm}$En cada columna, medias de grupos con letras iguales no son estadísticamente diferentes (Tukey, 0.05). 
Cuadro 4. Contenido promedio de proteína base en húmeda y en base seca, triptófano y lisina de 70 poblaciones de maíz de la Mixteca de Oaxaca de color pinto y variegado.

\begin{tabular}{|c|c|c|c|c|c|}
\hline Colecta & $\begin{array}{l}\text { Origen de la colecta (comunidad o agencia, } \\
\text { municipio) }\end{array}$ & $\begin{array}{l}\text { Proteína base } \\
\text { húmeda }(\%)\end{array}$ & $\begin{array}{l}\text { Proteína base } \\
\text { seca }(\%)\end{array}$ & $\begin{array}{l}\text { Triptófano } \\
\text { base seca }(\%)\end{array}$ & $\begin{array}{l}\text { Lisina base } \\
\text { seca }(\%)\end{array}$ \\
\hline \multicolumn{6}{|c|}{ Colectas de grano pinto y variegado } \\
\hline CIIDIR-150 & Benito Juárez, San Miguel El Grande & 9.0 & 10.1 & 0.052 & 0.353 \\
\hline CIIDIR-155 & Yuvitinaña, San Esteban Atatlahuca & 12.1 & 13.4 & 0.062 & 0.440 \\
\hline CIIDIR-156 & Yonama, San Esteban Atatlahuca & 11.4 & 12.8 & 0.075 & 0.460 \\
\hline CIIDIR-168 & Yucuiji, San Esteban Atatlahuca & 10.5 & 11.9 & 0.062 & 0.463 \\
\hline CIIDIR-171 & Independencia, San Esteban Atatlahuca & 13.0 & 14.6 & 0.069 & 0.340 \\
\hline CIIDIR-170 & Independencia, San Esteban Atatlahuca & 14.9 & 16.8 & 0.082 & 0.400 \\
\hline \multirow[t]{6}{*}{ CIIDIR-191 } & Yucuiji, San Esteban Atatlahuca & 12.5 & 13.8 & 0.069 & 0.350 \\
\hline & Promedio & $11.9 \mathrm{a}$ & $13.3 \mathrm{a}$ & $0.067 \mathrm{ab}$ & $0.400 \mathrm{a}$ \\
\hline & $\mathrm{DSH}^{\xi}$ & 1.90 & 2.13 & 0.008 & 0.230 \\
\hline & Testigo-QPM & 12.3 & 13.8 & 0.090 & 0.620 \\
\hline & $\mathrm{H}-564 \mathrm{C}^{\pi}$ & --- & --- & 0.086 & 0.352 \\
\hline & Maíces nativos de Nayarit" & --- & 10.3 & 0.050 & 0.304 \\
\hline
\end{tabular}

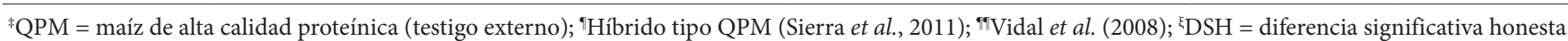
para la comparación de colectas (Tukey, 0.05).

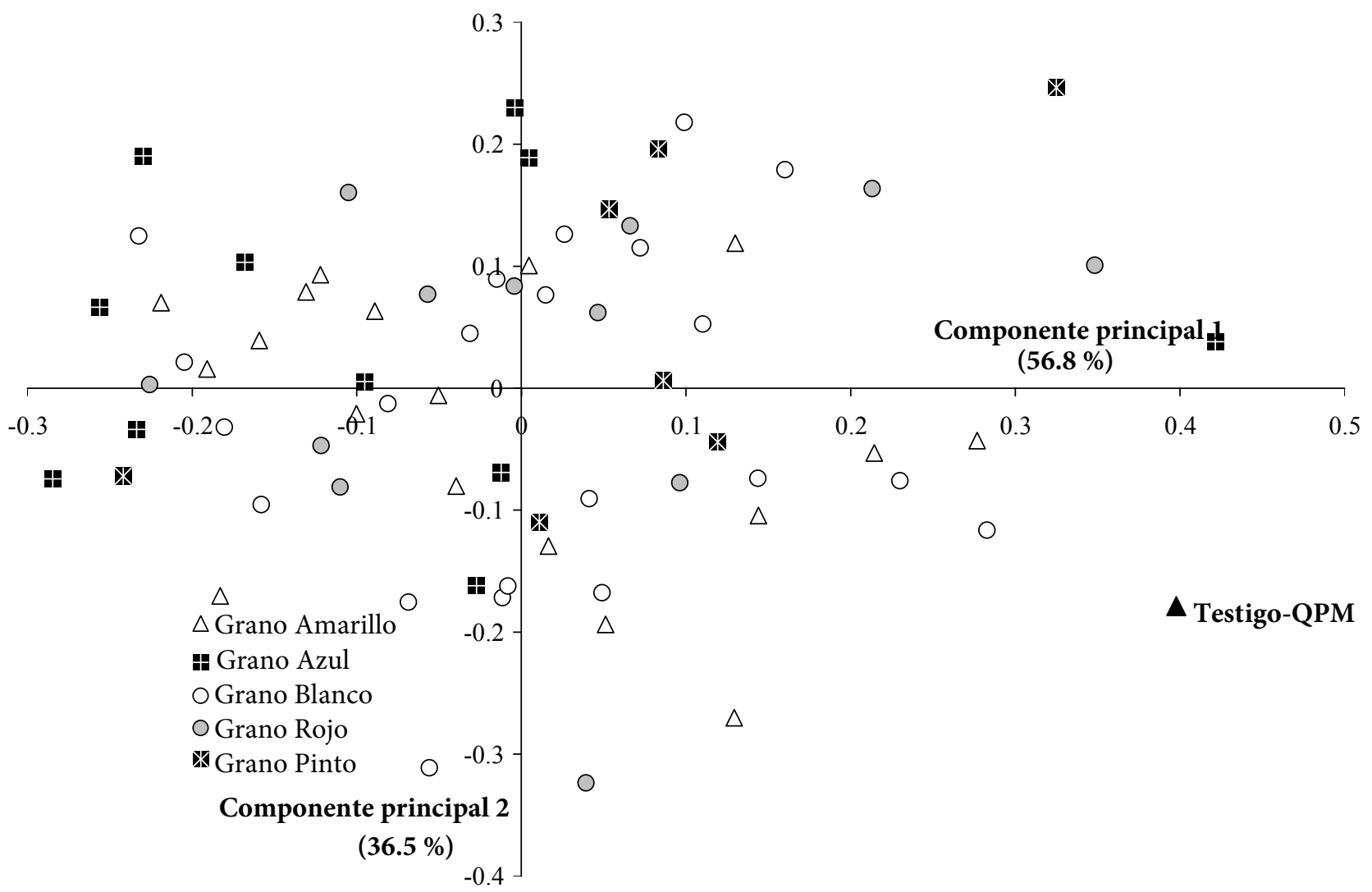

Figura 1. Dispersión de 70 poblaciones de maíz nativo colectado en la Mixteca Oaxaqueña, con base en el plano formado por los dos primeros componentes principales. 


\section{BIBLIOGRAFÍA}

Anderson E, J J Finan (1945) Maize in the Yanhuitlan codex. Ann. Mo. Bot. Gard. 32:361-368.

Benz B F (1997) Diversidad y distribución prehispánica del maíz mexicano. Arqueol. Mex. 5:16-23.

Bradford M M (1976) A rapid and sensitive method for the quantitation of microgram quantities of protein utilizing the principle of proteindye binding. Analyt. Biochem. 72:248-254.

Chávez S J L, P Diego F (2011) Familias campesinas y variación fenotípica de poblaciones nativas de maíz en la región de Tlaxiaco, Oaxaca. Des. Amb. Cult. 1:28-38.

Chávez-Servia J L, P Diego-Flores, J C Carrillo-Rodríguez (2011) Complejos raciales de poblaciones de maíz evaluadas en San Martín Huamelúlpam, Oaxaca. Ra Ximhai 7:107-115.

Contreras-Hinojosa J, V Volke-Haller, J Oropeza-Mota, C RodríguezFranco, T Martínez-Saldaña, A Martínez-Garza (2005) Reducción del rendimiento de maíz por la erosión del suelo en Yanhuitlán, Oaxaca, México. Terra Latinoam. 23:399-408.

Coutiño E B, G Vázquez C, B Torres M, Y Salinas M (2008) Calidad de grano, tortilla y botanas de dos variedades de maíz de la raza Comiteco. Rev. Fitotec. Mex. 31(Núm. Esp. 3):9-14.

Hagiwara A, K Miyashita, K Nakanishi, M Sano, S Tamano, T Kadota, T Koda, M Nakamura, K Imaida, N Ito, T Shirai (2001) Pronounced inhibition by a natural anthocyanin, purple corn color of 2-amino-1-methyl-6-phenylimidazol [4,5-b]pyridine (PhIP)associated colorectal carcinogenesis in male F344 rats pretreated with 1,2-dimethylhydrazine. Cancer Lett. 171:17-25.

Hayano-Kanashiro C, C Calderón-Vázquez, E Ibarra-Laclette, L Herrera-Estrella, J Simpson (2009) Analysis of gene expression and physiological responses in three Mexican maize landraces under drought stress and recovery irrigation. PLOS ONE 4:e7531. doi:10.1371/journal.pone.0007531.

López-Martínez L X, R M Oliart-Ros, G Valerio-Alfaro, C-H Lee, K L Parkin, H S García (2009) Antioxidant activity, phenolic compounds and anthocyanins content of eighteen strains of Mexican maize. LWT- Food Sci. Technol. 42:1187-1192.

Lozano-Alejo N, G Vázquez C, K Pixley, N Palacios-Rojas (2007) Physical properties and carotenoid content of maize kernels and its nixtamalized snacks. Innov. Food Sci. Emerg. Technol. 8:385-389.
Mittelmann A, J Branco de Miranda F, L Lourenço N, G J Melo M, C HaraKlein, R Machado S (2011) Quantitative variation for grain quality in Brazilian maize populations. Sci. Agric. (Piracicaba, Braz.) 68:50-56.

Muñoz O A, G Pérez J, P A López, R J Salvador (2002) Maíz de cajete: agrosistema y resistencia a sequía. In: Antología sobre Pequeño Riego, Vol. III. Sistemas de Riego no Convencionales. J Palerm (ed). Colegio de Postgraduados, Texcoco, México. pp:137-164.

OEIDRUS, Oficina Estatal de Información para el Desarrollo Rural Sustentable (2010) Anuario Estadístico de la Producción Agrícola 2010, Oaxaca. Disponible en: http://www.oeidrus-portal.gob.mx/ oeidrus_oax/a1.php (Diciembre 2011).

Obi I U (1982) Application of 2,4,6-trinitrobenzene-1-sulfonic acid (TNBS) method for determination of available lysine in maize seed. Agric Biol. Chem. 46:15-20.

Pressoir G, J Berthaud (2004) Population structure and strong divergent selection shape phenotypic diversification in maize landraces. Heredity 92:95-101.

Salinas-Moreno Y, F Martínez-Busto, M Soto-Hernández, R Ortega-Paczka, J L Arellano-Vázquez (2003) Efecto de la nixtamalización sobre las antocianinas del grano de maíces pigmentados. Agrociencia 37:617-628.

SAS Institute (1999) SAS Procedure Guide, Release 8.0. Institute Inc. Cary North Carolina, USA. $1643 \mathrm{p}$

Sierra M M, A Palafox C, F Rodríguez M, A Espinosa C, G Vázquez C, N Gómez M, S Barrón F (2011) H564C, híbrido de maíz con alta calidad de proteína para el trópico húmedo de México. Rev. Mex. Cien. Agríc. 2:71-84.

Soleri D, D A Cleveland (2001) Farmers' genetic perceptions regarding their crop populations: an example with maize in the Central Valleys of Oaxaca, Mexico. Econ. Bot. 55:106-128.

Vázquez M G, J P Pérez C, J M Hernández C, M L Marrufo D, E Martínez $\mathbf{R}$ (2010) Calidad de grano y de tortillas de maíces criollos del altiplano y valle del Mezquital México. Rev. Fitotec. Mex. 33(Núm. Esp. 4):49-56.

Vidal M V A, G Vázquez C, B Coutiño E, A Ortega C, J L Ramírez D, R Valdivia B, M J Guerrero H, F J Caro V, O Cota A (2008) Calidad proteínica en colectas de maíces criollos de la Sierra de Nayarit, México. Rev. Fitotec. Mex. 31(Núm. Esp. 3):15-21.

Villegas E, E Ortega, R Bauer (1985) Métodos químicos usados en el CIMMYT para determinar la calidad de la proteína de los cereales. Boletín Técnico 20. Centro Internacional de Mejoramiento de Maíz y Trigo. El Batán, Edo. de México. 32 p. 